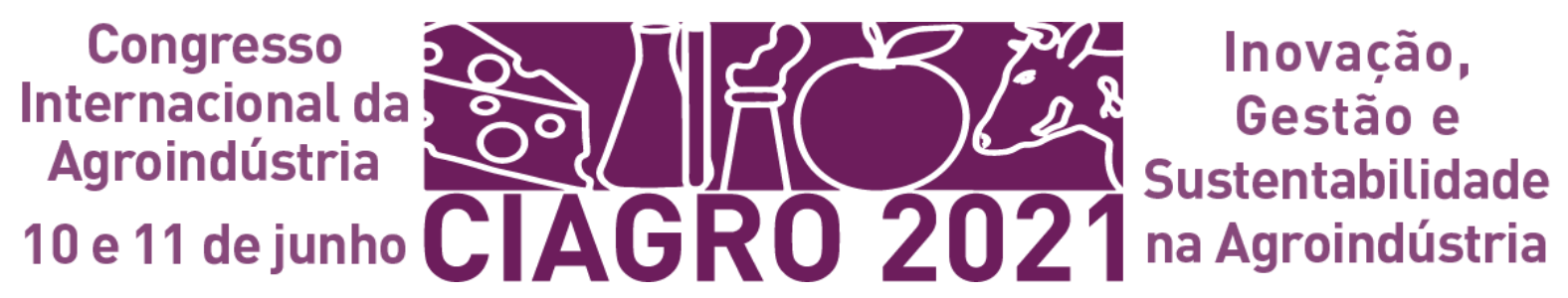

\title{
AVALIAÇÃO DO PROCESSAMENTO NOS COMPOSTOS FENÓLICOS E NA CAPACIDADE ANTIOXIDANTE EM ALHO NEGRO (ALLIUM SATIVUM)
}

\author{
EVALUACIÓN DEL PROCESAMIENTO EN COMPUESTOS FENÓLICOS Y \\ CAPACIDAD ANTIOXIDANTE EN AJO NEGRO (ALLIUM SATIVUM)
}

\author{
EVALUATION OF PROCESSING IN PHENOLIC COMPOUNDS AND \\ ANTIOXIDANTE CAPACITY IN BLACK GARLIC (ALLIUM SATIVUM)
}

\begin{abstract}
Diego Aires da Silva ${ }^{1}$; Jéssica Moreira Ferreira ${ }^{2}$; Brenda Moreira Lima ${ }^{3}$; Lorena Beatriz Figueiredo Viana ${ }^{4}$ Giselle Cristine Melo Aires ${ }^{5}$
\end{abstract}

DOI: https://doi.org/10.31692/IICIAGRO.0267

\begin{abstract}
RESUMO
O alho (Allium sativum) apresenta excelente valor nutricional, por conter em sua composição vitaminas, aminoácidos, enzimas, compostos biologicamente ativos e minerais. Seus diversos benefícios à saúde são geralmente atribuídos à presença de compostos organosulfurados, que também são responsáveis pelo odor e sabor característicos do alho. No entanto, possui utilização limitada devido ao seu sabor e aroma pungente. Nesse contexto, o alho negro vem ganhando espaço no mercado, um produto obtido a partir da maturação do alho por períodos prolongados, com temperatura e umidade relativa controladas. Este processo confere cor negra, sabor adocicado e aroma frutado, sem a pungência característica do alho cru. A literatura ainda é escassa em relação à sua produção e composição. Neste sentido, o presente trabalho visa sugerir uma nova metodologia de produção do alho negro, avaliar o processamento nos compostos fenólicos e na capacidade antioxidante, além de avaliar a características físico-química deste produto. Os resultados evidenciaram que o processamento térmico a $60^{\circ} \mathrm{C}$ por 15 dias em panela elétrica, teve efeito positivo nas características do produto em relação ao alho in natura nos parâmetros avaliados de umidade $(24,71 \%), \mathrm{pH}(4,6)$, açúcares redutores $(25,44 \mathrm{~g} \cdot 100 \mathrm{~g}-1$ de matéria seca) e com incremento nos conteúdos de compostos fenólicos $(28,07 \mathrm{mg}$ EAG/g) e capacidade antioxidante $(19,89 \mu \mathrm{M}$ Trolox/g). O alho negro obtido no presente trabalho apresentou diferença significativa $(p<0,05)$ em relação ao comercial, para as características físicas e químicas avaliadas, exceto para o valor de $\mathrm{pH}$.
\end{abstract}

Palavras-Chave: Allium sativum, maillard, alho negro

\footnotetext{
${ }^{1}$ Tecnologia de Alimentos, Universidade do Estado do Pará diegoaires@ uepa.br

2 Tecnologia de Alimentos, Universidade do Estado do Pará jessicaferreira1142@ gmail.com

${ }^{3}$ Tecnologia de Alimentos, Universidade do Estado do Pará moreirabrenda433@ gmail.com

${ }^{4}$ Tecnologia de Alimentos, Universidade do Estado do Pará d.lorevianna@ gmail.com

${ }^{5}$ Tecnologia de Alimentos, Universidade do Estado do Pará giselle.aires@uepa.br
} 


\section{ABSTRACT}

Garlic (Allium sativum) has an excellent nutritional value, as it contains vitamins, amino acids, enzymes, biologically active compounds and minerals. Its various health benefits are generally attributed to the presence of organosulfur compounds, which are also responsible for the characteristic odor and flavor of garlic. However, it has limited use due to its pungent flavor and aroma. In this context, black garlic has been gaining space in the market, a product obtained from the maturation of garlic for prolonged periods, with controlled temperature and relative moisture. This process gives it a black color, a sweet taste and a fruity aroma, without the pungency characteristic of raw garlic. Literature is still scarce in relation to its production and composition. In this sense, the present work aims to suggest a new methodology for the production of black garlic, to evaluate the processing of phenolic compounds and antioxidant capacity, in addition to evaluating the physical and chemical characteristics of this product. The results showed that the thermal processing at $60^{\circ} \mathrm{C}$ for 15 days in an electric pan, had a positive effect on the characteristics of the product in relation to garlic in natura in the evaluated parameters of humidity (24.71\%), pH (4.6), reducing sugars (25.44 g $100 \mathrm{~g}-1$ of dry matter) and with an increase in the contents of phenolic compounds (28.07 $\mathrm{mg}$ EAG / g) and antioxidant capacity (19.89 $\mu \mathrm{M}$ Trolox / $g)$. The black garlic obtained in the present work showed a significant difference $(p<0.05)$ in relation to the commercial one, for the physical and chemical characteristics evaluated, except for the $\mathrm{pH}$ value.

Keywords: Black garlic. Garlic Processing. Antioxidant activity. Phenolic Compounds

\section{INTRODUÇÃO}

O alho (Allium sativum L.) é definido como uma especiaria, produto constituído de partes (raízes, rizomas, bulbos, cascas, folhas, flores, frutos, sementes e talos) de uma ou mais espécies vegetais, tradicionalmente utilizadas para agregar sabor ou aroma aos alimentos e bebidas (BRASIL, 2005). É uma espécie cultivada há milhares de anos, tendo as zonas temperadas da Ásia Central como seu principal centro de origem, se espalhando para a região do Mediterrâneo (VIEIRA, 2004).

Esta hortaliça possui importância sócio-econômica no Brasil, por ser cultivada, em sua grande maioria por pequenos produtores, com utilização da mão de obra familiar (VIEIRA, 2004; SILVA, LEITE \& RESENDE, 2008), em várias regiões do país, apresentando uma maior produtividade nos estados de Minas Gerais, Goiás, Santa Catarina e Rio Grande do Sul (CONAB, 2020).

O alho apresenta um valor energético de $121 \mathrm{kcal}$ por $100 \mathrm{~g}$, devido ao elevado teor de carboidratos $(25,8 \mathrm{~g})$ e proteínas $(5,70 \mathrm{~g})$, além de um excelente valor nutricional por conter em 
sua composição vitaminas do complexo B e minerais (TBCA, 2020).

O alho é considerado um alimento funcional rico em alicina, possui ação antiviral, antifúngica, antibiótica e antioxidante (BUTT et al., 2009; CONCEIÇÃO, 2013; FONSECA et al., 2014; SANTHOSHA; JAMUNA \& PRABHAVATHI, 2013), e apresenta também em sua composição o ácido fosfórico, ácido sulfúrico, proteínas e sais minerais. Além de compostos fenólicos e organosulfurados responsáveis tanto pelo odor e sabor, quanto pela ação antioxidante (CORZO-MARTINEZ, CORSO \& VILLAMIEL, 2007; SILVA, 2013). No entanto, o uso de alho cru é limitado, devido ao seu sabor e aroma pungente, nesse contexto, surge a necessidade de novos processamentos e produtos no mercado que venham reduzir essa adstringência, mantendo ou potencializando os benefícios e que apresente vantagens comerciais.

O alho negro é obtido a partir do processamento térmico do alho fresco (Allium sativum L.), sob temperatura e umidade relativa controladas por um determinado período. Através da reação de Maillard os dentes de alho se tornam escuros, com sabor adocicado e mudanças de textura (YUAN, 2018).

Diversos estudos relatam que o alho negro apresenta variadas funções, como efeitos antioxidantes, antialérgicos, antidiabetes, anti-inflamatórios e anticarcinogênicos (CORZOMARTINEZ, CORSO \& VILLAMIEL, 2007; PEREIRA et al. 2018; ZOU et al., 2017). Portanto, o alho negro reduz a pungência sensorial característica desta matéria prima, e seu consumo se mostra benéfico a saúde (PIRES, 2014).

Nesse contexto, este trabalho tem como objetivo produzir o alho negro e avaliar suas características físico-químicas, capacidade antioxidante e compostos fenólicos.

\section{REFERENCIAL TEÓRICO}

\subsection{Alho}

Entende-se por alho o bulbo da espécie Allium sativum, L. (Figura 1) que se apresenta com as características do cultivar bem definidas, fisiologicamente desenvolvido, inteiro, sadio e isento de substâncias nocivas à saúde (BRASIL,1992). É uma planta pertencente à família Liliacea, que apresenta mais de 700 espécies, incluindo a cebola, o alho-poró e a cebolinha (NOVO, 2018).

A produção de alho no Brasil em 2018, foi estimada em 118,8 mil toneladas (IBGE, 2018). Esse cultivo apresenta grande importância, tanto pela área plantada, quanto pela geração de mão de obra, mas essa cultura vem perdendo espaço devido à importação (PIRES, 2014). 
Em 2019 foram importados um total de 165,4 mil toneladas, sendo 77,7 mil toneladas da Argentina e 58,4 mil toneladas da China que é o maior fornecedor de alho para o Brasil. Outros países complementam o total importado (CONAB, 2020).

A comissão técnica de normas e padrões do Ministério da Agricultura, Pecuária e Abastecimento, classifica o alho em dois grupos: o branco e o roxo, de acordo com a coloração da película do bulbilho. E em dois subgrupos de acordo com o número de bulbilhos por bulbo: o nobre, que apresenta de 5 a 20 bulbilhos e o comum com mais de 20 bulbilhos (BRASIL,1992). Da planta, para o consumo, o bulbo (cabeça) é o mais utilizado, este é dividido em bulbilhos (dentes), onde se armazenam os componentes aromáticos e nutricionais do alho (PEREIRA et al. 2018).

O alho tem excelente valor nutricional, por conter em sua composição vitaminas, aminoácidos, enzimas, compostos biologicamente ativos e minerais. (SILVA, 2013; TACO, 2011). Sendo amplamente utilizado não só para fins culinários, mas também medicinais (FONSECA et al., 2014; LI, XU \& SUN, 2007; RÍOS-RÍOS et al., 2019). Apresenta potencial antioxidante, que é atribuído aos compostos alilo e polifenólicos. Pela ação da enzima alinase, a alina na presença de água é convertida nas substâncias alicina (Dialil thiosulfinate) e Salilcisteína (SAC), estas são responsáveis pelo odor pungente caraterístico do alho (MALDONADE \& MACHADO, 2016).

Sua principal função, em termos culinários é fornecer o seu aroma e sabor característico aos alimentos, sendo utilizado picado, esmagado, cru, frito ou cozido, com o intuito de temperar carnes, molhos entre outros tipos de alimentos. Além de ser encontrado no mercado na forma pulverizada, líquida, pasta, óleo ou extratos (CONCEIÇÃO, 2013; MALDONADE \& MACHADO, 2016).

Por apresentar um sabor pungente característico, o consumo do alho cru não é muito difundido, levando ao estudo e utilização de novos processos industriais, tais como envelhecimento, fermentação e tratamento térmico para a obtenção de produtos de alho com redução do odor pungente e que mantenha as suas propriedades benéficas (BOTAS, 2017; TOLEDANO-MEDINA et al., 2016).

\subsection{Alho negro}

\subsubsection{Processo de produção}

O alho preto ou alho negro (Figura 1), é obtido a partir do alho in natura, maturação em um determinado período de tempo por alta temperatura, alterando sua composição química, textura, cor, sabor e, em relação ao alho fresco, não libera odor desagradável, pois tem teor de 
alicina reduzido, por ser um composto instável quando submetido à elevadas temperaturas. Sua origem é desconhecida, mas países como Japão, Coreia do Sul e Tailândia já consumiam há muitos anos, e nos últimos 10 anos seu consumo vêm crescendo e ganhando espaço na culinária de vários países (KIMURA, 2017; MALDONADE \& MACHADO, 2016).

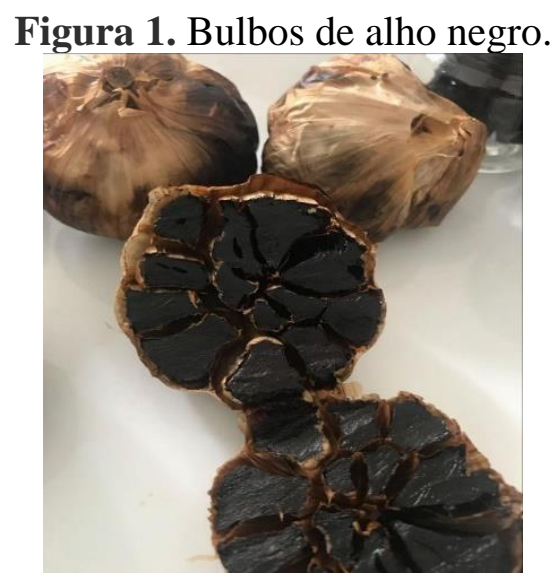

Fonte: Autor

Existem várias patentes para o processo de produção do alho negro, as quais diferem-se em relação ao período de processamento, que pode variar de 7 à 60 dias; temperatura (60-90 ${ }^{\circ} \mathrm{C}$ ), umidade relativa controlada por meio de soluções salinas saturadas (70-90\%), tipo de recipiente usado para a maturação, além do uso de fermentação nos processos em temperaturas mais baixas (BOTAS, 2017). Após esse processo o novo produto é apresentado com características diferentes, com cor preta, cheiro agradável e uma doçura de fruta, podendo este ser consumido em seguida (BAE et al., 2014; NODA et al., 2013; PIRES \& MAURO, 2016; SILVA, 2013).

Podem ser utilizados equipamentos de larga escala na produção de alho negro, tais como estufa-secador e câmaras de fermentação com diferentes capacidades de produção. Por ter uma demanda de tempo de produção em torno de 30-60 dias em temperatura elevada e alta umidade, torna-se necessário não apenas reduzir o tempo de procedimento, mas tornar menos complexo o processo de produção (NODA et al., 2013).

\subsubsection{Composição do alho negro e suas funcionalidades}

O processo de envelhecimento do alho negro está diretamente ligado a reação de Maillard devido a formação de melanoidinas responsáveis por promover a coloração negra ao alho, em pH favorável de 6 e 8 (PIRES, 2014; SHIBAO \& BASTOS, 2011). O produto possui um alto teor de polissacarídeos, proteínas, compostos fenólicos e compostos orgânicos de enxofre (LU et al., 2017). Pesquisas mostram que alguns componentes do alho negro ajudam 
no combate de doenças, inibição do crescimento da linha celular cancerosa, atividade antiobesidade, ação antialérgica e também na redução de lipídios no sangue (KIMURA et al., 2017; PIEKARSKI, 2013).

Lu et al. (2017), destacaram em seu estudo a elevada atividade antioxidante do alho negro, e um aumento no conteúdo de polifenóis e flavonoides durante o período de aquecimento. Ademais, compostos orgânicos de enxofre, alcaloides da $\beta$-carbolina e melanoidinas são encontrados em abundância neste produto (ZOU et al., 2017).

A inserção do alho negro na dieta pode melhorar ou reduzir a gordura corporal, baixar a hiperlipidemia e proteger contra o estresse, também previne complicações diabéticas, e é usado como antioxidante para proteger as células de doenças e assim retardar o envelhecimento delas (SILVA, 2013).

O alho negro é utilizado a centenas de anos nos países orientais, especialmente na Coréia, devido as suas propriedades medicinais e também por sua versatilidade culinária, sendo utilizado nas preparações de purês, molhos ou adicionados às massas, arroz, pizzas, inteiros ou picados. Por apresentar um sabor único, sua popularidade se espalhou pelo mundo e recentemente foi introduzido a alta gastronomia ocidental (MALDONADE \& MACHADO, 2016; PEREIRA et al. 2018).

\subsection{Reação de Maillard}

A reação de Maillard, corresponde a uma série de reações de escurecimento não enzimático, que surgem principalmente durante o aquecimento e armazenamento prolongado de produtos alimentícios, no qual ela confere influência à atributos sensoriais fundamentais para aceitação de alimentos termicamente processados, com a geração de compostos voláteis responsáveis pelo sabor, aroma, cor e mudança de textura (BASTOS et al., 2011; SHIBAO, 2010). Esta reação é subdividida em três estágios: estágio inicial, estágio intermediário e estágio final (BASTOS et al., 2011; BRIÃO et al., 2011; LIU et al., 2014).

$\mathrm{Na}$ primeira etapa ocorre a condensação entre um açúcar redutor e um grupamento amino, formando os produtos do rearranjo de Amadori (açúcar aldose) ou levando aos produtos de Heyns (açúcar cetose). Os compostos formados levam ao início da etapa intermediária, ocorrendo a fragmentação dos compostos de Amadori e a formação de substâncias de coloração amarelada com absorção intensa na região ultravioleta. No estágio final, há a desidratação, ciclização e reações de polimerização, formando pigmentos poliméricos de coloração marrom, como as melanoidinas. Estes pigmentos levam à coloração dos alimentos, sendo desejáveis ou 
indesejáveis e fazendo parte do estágio final da reação de Maillard (FRANCISQUINI et al., 2017; LIU et al., 2014; SHIBAO, 2010).

A ocorrência da reação de maillard está relacionada a vários fatores, como temperatura (acima de $40{ }^{\circ} \mathrm{C}$ ), atividade de água $(0,4$ a 0,7$), \mathrm{pH}$ (6 a 8), umidade relativa (30\% a $70 \%$ ) e a reação pode ser catalisada na presença de íons metálicos de transição como $\mathrm{Cu}^{2+}$ e $\mathrm{Fe}^{2}$ e pela composição química do alimento (KWAK \& LIM, 2004; PIRES \& MAURO, 2016; RODRIGUEZ et al., 2016; YILMAZ \& TOLEDO, 2005).

\section{METODOLOGIA}

\subsection{Matéria-prima}

As amostras de alhos (Allium Sativum L.) frescos foram selecionadas do grupo de alho roxo e subgrupo nobre e foram adquiridas em um mercado local da cidade de Cametá - Pará, localizada à margem esquerda do Rio Tocantins. Os bulbos ficaram armazenados à temperatura ambiente em local fresco, até utilização nos experimentos. $\mathrm{O}$ alho negro comercial foi cedido por uma empresa localizada na cidade de Belém-PA.

\subsection{Processamento}

Para a produção de alho negro, uma panela elétrica (Figue 2) da marca Funkitchen com capacidade de $3 \mathrm{~L}$, teve seu interior forrado com papel manteiga, onde foram colocados 10 bulbos de alho, conforme pode ser observado na figura 4. Em seguida, acoplou-se um termômetro na saída de vapor para controle da temperatura em $60{ }^{\circ} \mathrm{C}$. Após 15 dias de processo, ver figura 6 , os bulbos foram retirados e armazenados em embalagens de polipropileno sob vácuo à temperatura ambiente e abrigado de luz.

Figura 2. Panela elétrica contendo os alhos em início de processo e alho pós processamento.

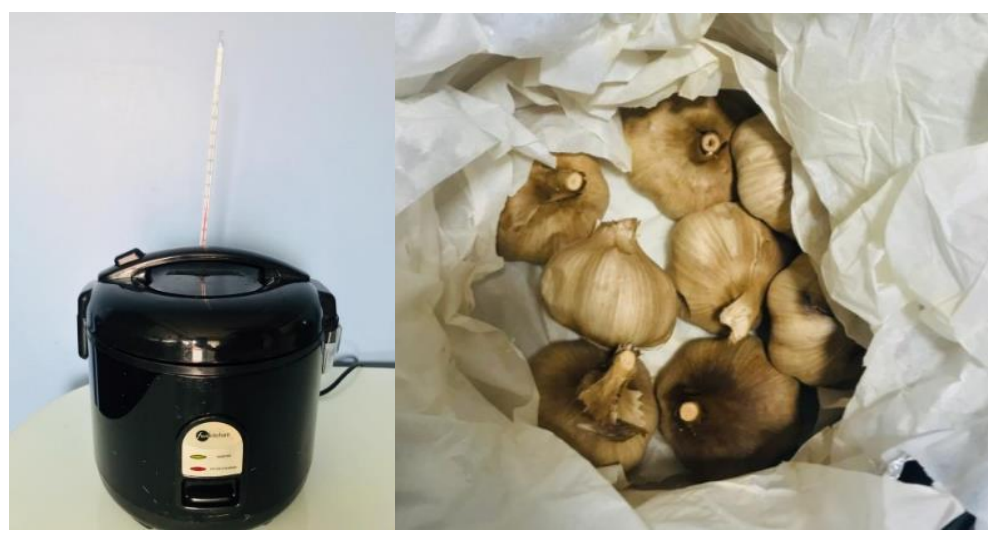

Fonte: Autor. 
4.3 Análises físico-químicas no alho in natura e no alho negro

Análises de umidade, sólidos solúveis, $\mathrm{pH}$, açúcares redutores e totais, compostos fenólicos totais e capacidade antioxidante, foram realizadas nas amostras de alho in natura e de alho negro artesanal e comercial, em caráter de comparação.

\subsubsection{Umidade}

A umidade foi determinada nas amostras de alho in natura, alho negro comercial e alho negro artesanal. A determinação da umidade foi realizada seguindo a metodologia de dessecação em estufa a $105^{\circ} \mathrm{C}$, descrita por ADOLFO LUTZ (2008).

\subsubsection{Sólidos Solúveis}

Leitura em refratômetro analógico (AKROM- REF90, precisão, 1\%) com escala de 0 a $90 \%$ e compensação automática de temperatura (ATC) de 10 a $30{ }^{\circ} \mathrm{C}$, devidamente calibrado com água destilada.

\subsection{3 $\mathrm{pH}$}

Para análise de $\mathrm{pH}$ foi determinado através da imersão do eletrodo (Marconi) nas amostras diluídas em água destilada a $20^{\circ} \mathrm{C}$ (INSTITUTO ADOLFO LUTZ, 2008).

\subsubsection{Açúcares redutores e totais}

As análises foram realizadas pelo método titulométrico com soluções de Fehling A e B, de acordo com o Instituto Adolfo Lutz, 1985.

\subsubsection{Compostos fenólicos totais}

O teor de compostos fenólicos totais foi determinado pelo método descrito por Singleton e Rossi (1965), modificado por (GEORGÉ et al., 2005), o qual baseia-se na reação com o reagente de Folin-Ciocalteau. A quantificação foi feita em espectrofotômetro a $760 \mathrm{~nm}$, com as medições em triplicata para cada amostra. O conteúdo fenólico total foi calculado com base na curva padrão do ácido gálico e expresso em mg de ácido gálico equiv / g.

\subsubsection{Capacidade antioxidante}

A determinação de capacidade antioxidante foi realizada através do método ABTS, conforme metodologia proposta por Re et al. (1999), em que mede-se a diminuição de 
concentração do radical ABTS [ácido 2,2-azin9-bis (3-etilbenzotiazolin)-6-sulfônica] capturado pelo antioxidante testado da amostra e pelo equivalente hidrossolúvel da vitamina $\mathrm{E}$ (trolox). O extrato foi preparado a partir de aproximadamente 1 grama de amostra, utilizando como solvente extrator metanol a 50\%, acetona a 70\% e água destilada, o qual foi homogeneizado, centrifugado (11.000 rpm por 15 minutos) e filtrado em duas etapas, este filtrado foi transferido para um balão volumétrico e aferido para $50 \mathrm{~mL}$ com água destilada.

Para obtenção da curva padrão, as amostras foram analisadas em triplicata, após preparo e leitura do padrão de trolox $2 \mathrm{mM}$, em diferentes diluições. A leitura foi realizada em espectrômetro à $734 \mathrm{~nm}$ após 6 minutos da mistura do radical ABTS $(5 \mathrm{~mL}$ de solução de ABTS $7 \mathrm{mM}$ e $88 \mu \mathrm{L}$ de solução de persulfato de potássio $140 \mathrm{mM}$ ), com o extrato da amostra em diferentes diluições, utilizando o álcool etílico como branco.

\subsubsection{Análise estatística}

Os resultados avaliados foram expressos por análise de variância (ANOVA), por intervalo de confiança de $95 \%$ ( $\mathrm{p}<0,05$ considerados significativamente) com isso as médias foram comparados pelo teste Tukey, por meio do software versão 7.0 (STATSOFT INC., 2004).

\section{RESULTADOS E DISCUSSÃO}

4.4 Caracterização da Matéria-prima

Durante o processamento o alho adquiriu uma coloração preta, devido a reação de escurecimento não enzimático, que depende muito da temperatura de aquecimento, sendo ela um indicador de maturação do alho negro (ZHANG et al.; 2016).

Após 15 dias de incubação a $60{ }^{\circ} \mathrm{C}$ do alho in natura foi obtido um alho de cor negra, sem odor característico de alho, macio e casca de coloração dourada, como observado na Figura 3.

Figura 3. Alho negro obtido após 15 dias de processamento.

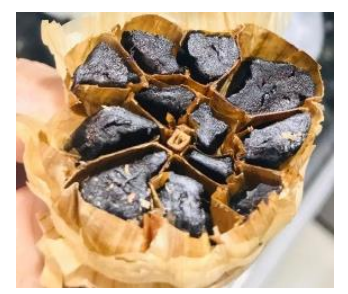

Fonte: Autor. 
Como observa-se na Tabela 1, as amostras de alho in natura, negro comercial e artesanal diferiram estatisticamente $(\mathrm{p}<0,05)$ para os valores de umidade, sólidos solúveis, açúcar total, redutor e não redutor.

Tabela 1 - Caracterização do alho in natura (AI), alho negro comercial (AC) e alho artesanal (AA).

\begin{tabular}{cccc}
\hline Análise & AI & AC & AA \\
\hline Açucares Totais & $34,63 \pm 0,52^{\mathrm{a}}$ & $41,03 \pm 0,73^{\mathrm{b}}$ & $65,88 \pm 1,84^{\mathrm{c}}$ \\
Açucar Redutor & $\mathrm{ND}$ & $21,27 \pm 0,34^{\mathrm{a}}$ & $25,44 \pm 0,55^{\mathrm{b}}$ \\
Açucar Não Redutor & $34,63 \pm 0,52^{\mathrm{a}}$ & $19,76 \pm 0,81^{\mathrm{b}}$ & $40,44 \pm 1,29^{\mathrm{c}}$ \\
Umidade & $65,76 \pm 0,13^{\mathrm{a}}$ & $28,56 \pm 0,5^{\mathrm{b}}$ & $24,71 \pm 0,02^{\mathrm{c}}$ \\
Sólidos Solúveis Totais & $13,17 \pm 0,29^{\mathrm{a}}$ & $21,83 \pm 0,29^{\mathrm{b}}$ & $27,33 \pm 0,29^{\mathrm{c}}$ \\
pH & $6,6 \pm 0,00^{\mathrm{a}}$ & $4,6 \pm 0,00^{\mathrm{b}}$ & $4,67 \pm 0,06^{\mathrm{b}}$
\end{tabular}

Letras diferentes na horizontal diferem significativamente $(\mathrm{p}<0,05)$. Médias em triplicata + DP. ND: Não detectado.

Neste estudo, foram encontrados valores de umidade para o alho negro comercial e o alho negro artesanal, semelhantes aos encontrados por Silva (2013) e valores menores que o relatado por Choi, Cha, Lee (2014) que encontraram uma umidade de $31,77 \%$ no $14^{\circ}$ dia de análise do alho negro a $70^{\circ} \mathrm{C}$ com $90 \%$ UR.

Para o teor de sólidos solúveis do alho negro artesanal, o resultado encontrado neste trabalho foi de $27,33{ }^{\circ}$ Brix, um resultado superior ao encontrado por Maldonade e Machado (2016) $\left(21,10^{\circ}\right.$ Brix) ao analisarem SST de alho negro comercial e alho negro produzido pela Embrapa.

O processo de envelhecimento do alho promoveu aumento dos teores de sólidos solúveis totais (SST), tanto na amostra comercial, quanto nos bulbos processados neste estudo. Este resultado pode estar relacionado com a doçura característica do alho negro.

$\mathrm{O}$ valor de pH observado para as amostras de alho in natura foi de 6,60 e para o alho negro artesanal de 4,67 (Tabela1). Toledano-Medina et al., (2016) ao avaliarem a evolução de algumas propriedades físico-químicas e antioxidantes de bulbos de alho negro, observaram o valor de $\mathrm{pH}$ de 6,31 para o in natura e 4,87 para o negro obtido a $72^{\circ} \mathrm{C}$ por 11 dias. A diminuição do $\mathrm{pH}$ ocorre durante o processo de transformação do alho in natura em alho negro com o aumento do tempo de processamento térmico e temperatura utilizados (BAE et al., 2014; YUAN et al., 2018).

Os açúcares não redutores inicialmente presentes no alho in natura foram, em boa parte hidrolisados, sendo transformados em açúcares redutores. O pH inicial de 6,60 sofreu redução 
durante o processo, propiciando uma hidrólise. Segundo Dalonso et al. (2009) a formação de açúcares redutores pode ocorrer via hidrólise ácida, e não enzimática, uma vez que os oligossacarídeos (inulina) presentes no alho podem ser hidrolisados em condições ácidas, possibilitando um aumento no conteúdo de açúcares.

As amostras de alho negro testadas, também foram caracterizadas por um conteúdo diferenciado $(p<0,05)$ de açúcares (Tabela 1). O conteúdo médio de açúcares redutores no alho in natura não foi detectado, de modo que, no alho negro artesanal este valor, foi significativamente maior $(25,44 \pm 0,55 \mathrm{~g} \cdot 100 \mathrm{~g}-1$ de matéria seca) do que no alho comercial $(21,27 \pm 0,34 \mathrm{~g} \cdot 100 \mathrm{~g}-1 \mathrm{de}$ matéria seca). Os resultados para alho negro, obtidos neste estudo, são semelhantes aos dados obtidos por Pires, (2014) ao avaliar o alho negro a $65^{\circ} \mathrm{C}$. Assim como neste estudo, Pires \& Mauro (2016) observaram que há um aumento do teor de açúcares redutores ao final do processamento do alho negro.

\subsection{Compostos fenólicos e capacidade antioxidante}

Os valores encontrados referentes a caracterização de compostos fenólicos e atividade antioxidante estão expressos na Tabela 2.

Tabela 2 - Valores médios de compostos fenólicos (mg EAG/g) e atividade antioxidante ( $\mu \mathrm{M}$ Trolox/g) do alho in natura (AI), alho negro comercial (AC) e alho artesanal (AA).

Análise

\section{Compostos Fenólicos}

Atividade Antioxidante
AI

$$
0,99 \pm 0,05^{\mathrm{a}}
$$

$1,83 \pm 0,05^{\mathrm{a}}$
$\mathrm{AC}$
AA

$28,07 \pm 1,06^{\mathrm{c}}$

Letras diferentes na horizontal diferem significativamente $(\mathrm{p}<0,05)$.

Médias em triplicata + DP.

Jang, Seo \& Lee (2008), realizaram a determinação de compostos polifenólicos (10 $\mathrm{mg} / \mathrm{g}$ ) em extratos de alho negro e alho in natura e constataram que há um aumento significativo no teor desse bioativo do alho negro em comparação ao alho in natura, assim como nos estudos realizado por Pires, (2020) (4,92mg/g).

Os valores determinados de compostos fenólicos totais nos alhos negros processados neste estudo foram cerca de 3 vezes maiores do que aqueles encontrados na amostra comercial e muito superior ao in natura. Sendo esse crescimento é semelhante ao encontrado por Kim, Kang \& Gweon (2013) ao compararem os teores de ácidos fenólicos e flavonoides em diferentes etapas do processamento térmico.

O aumento do conteúdo de compostos fenólicos no alho negro pode ser justificado pela perda de água e também pela formação de compostos fenólicos no processo (WANG \& SUN, 
2017). Os compostos instáveis no alho cru são transformados em compostos solúveis estáveis com alto poder antioxidante durante o tratamento térmico (CORZO-MARTINEZ, CORSO \& VILLAMIEL, 2007). Estudos realizados com alho negro destacam que o aumento de sua capacidade antioxidante pode ser pela crescente no teor de polifenóis e S-alil-cisteína, um composto derivado da alina (LEE et al., 2009; NAJMAN, SADOWSKA \& HALLMANN, 2020).

Como ocorrido com CF, o aumento da atividade antioxidante no alho negro se deve não somente à perda de água, mas também à formação de compostos antioxidantes, como mostra a Tabela 2. Ao final do tratamento térmico observou-se um aumento da capacidade antioxidante de 10 vezes mais no alho negro artesanal, sendo significativamente maior do que no alho in natura. Lee et al. (2009), descreveram um aumento de 6,5 vezes na capacidade antioxidante do alho negro, fator também observado por Toledano-Medina et al. (2016).

A capacidade antioxidante do alho aumenta com o aumento da temperatura do processo (TOLEDANO-MEDINA et al., 2016), uma relação semelhante à mostrada por Choi, Cha, Lee (2014) durante o tratamento térmico de alho negro a $70{ }^{\circ} \mathrm{C}$, em que notaram um aumento significativo $(p<0,05)$ do teor de componentes antioxidantes, confirmando a relação positiva entre as propriedades antioxidantes do alho negro e temperatura do tratamento térmico.

\section{CONSIDERAÇÕES FINAIS}

O tratamento térmico com umidade controlada realizado em panela elétrica confe ao alho características sensoriais característicos do produto. Constata-se uma alta redução do teor de umidade após o tratamento, apresentando valores similares a literatura. $\mathrm{O}$ alho negro artesanal apresentou um aumento no teor de sólidos solúveis e alteração da composição de açúcares durante o processo devido à hidrólise de açúcares não redutores.

Com relação à capacidade antioxidante do alho negro, constatou-se aumento do conteúdo de seus compostos fenólicos e de sua atividade antioxidante em relação ao alho in natura, não somente devido à perda de água, mas também pela formação desses compostos.

O alho negro artesanal, em comparação ao alho negro comercial, apresentou diferença em relação às características físicas e químicas avaliadas, exceto para o valor de pH. Por fim, concluiu-se que 15 dias de processamento foram suficientes para a obtenção do alho negro, com teor de compostos fenólicos e índice de capacidade antioxidante expressivos. 


\section{REFERÊNCIAS}

ANGELO, P. M.; JORGE, N. Compostos fenólicos em alimentos - uma breve revisão. Rev. Inst. Adolfo Lutz (Impr.), São Paulo, v. 66, n. 1, 2007.

BAE, S. E; CHO, S. Y; WON, Y. D.; LEE, S. H.; PARK, H. J. Changes in S-allyl cysteine contents and physicochemical properties of black garlic during heat treatment. LWT - Food Science and Technology, Volume 55, 2014.

BARBOSA, T. N. R. M., FERNANDES. D. C. compostos bioativos e doenças cardiovasculares: revisando as evidencias científicas. Goiânia, v. 41, n. 2, p. 181-192. Jun. 2014.

BASTOS, D. H. M.; SHIBAO, J.; FERREIRA, E. L.; BOMBO, A. J. Produtos da reação de Maillard em alimentos. Nutrire: rev. Soc. Bras. Alim. Nutr.= J. Brazilian Soc. Food Nutr., São Paulo, SP, v. 36, n. 3, p. 63-78, dez. 2011.

BOTAS, J. C. S. Caracterização química e propriedades bioativas de Allium sativum L. com diferentes proveniências e processamentos. Bragança: IPB e US. 2017. 61p. (Dissertação mestrado).

BRASIL, MINISTÉRIO DA AGRICULTURA, PECUÁRIA E ABASTECIMENTO - MAPA (1992). Portaria RDC $n^{\circ}$ 242, de 17 de setembro de 1992. Norma de Identidade, Qualidade, Acondicionamento, Embalagem e Apresentação do Alho. Diário Oficial da União, Brasília, DF, 24 set. 1992.

BRASIL. Ministério da Saúde. Resolução RDC n. 276, de 22 de setembro de 2005. Regulamento técnico para especiarias, temperos e molhos. Diário Oficial [da] República Federativa do Brasil, Poder Executivo, Brasília, DF, 23 jun. 2005. Seção 1.

BRASIL. Ministério da Saúde e Anvisa. Monografia da espécie Allium Sativum (Alho). Brasília 2015. Disponível em: https://portalarquivos2.saude.gov.br/images/pdf/2017/setembro/11/MonografiaAllium.pdf. Acesso em: 26 de Jan. de 2020.

BRIÃO, V. B., FOLLMER, L.; SOUZA, M.; RODRIGUES, V. M. Cinética do escurecimento não-enzimático com soluções modelo de açúcares e aminoácidos em pH neutro e ácido. Acta Scientiarum Technology, v. 33, n. 1, p. 87-93, 2011.

BUTT M. S.; SULTAN M. T.; BUTT M. S.; IQBAL; J. Garlic: nature's protection against physiological threats. Crit Rev Food Sci Nutr. Jun;49(6):538-51, 2009.

CHOI, I. S.; CHA, H. S.; LEE, Y. S. Physicochemical and Antioxidant Properties of Black Garlic. Molecules 2014 , 19 (10), 16811-16823.

CONAB - Companhia Nacional de Abastecimento. Alho. Histórico mensal alho, período: Dezembro de 2019. (2020). Disponível em: https://www.conab.gov.br/info-agro/analisesdo-mercado-agropecuario-e-extrativista/analises-do-mercado/historico-mensal-de-alho. Acesso em: 23 de Jan. de 2020.

CONCEIÇÃO, S.F.S.M. Efeitos do Gengibre, do Alho e do Funcho na Saúde. 79 p. Dissertação de Mestrado - Universidade Fernando Pessoa, Faculdade Ciências da Saúde, Mestre em Ciências Farmacêuticas. Porto, 2013.

CORZO - MARTINEZ, M.; CORSO, N. \& VILLAMIEL, M. Propriedades biológicos das cebolas e alho. Trends Food Sci Technol. 18 : 609 - 625 (2007).

DALONSO, N.; IGNOWSKI, E.; MONTEIRO, C. M. A.; GELSLEICHTER, M.; WAGNER, T. M.; SILVEIRA, M. L. L.; SILVA, D. A. K. Extração e caracterização de carboidratos presentes no alho (Allium sativum L.): proposta de metodologia alternativa. Ciência e Tecnologia de Alimentos, v. 29, n. 4, p.793-797, 2009.

FONSECA, G.M.; PASSOS, T.C.; NINAHUAMAN, M.F.M.L.; CAROCI, A.S.; COSTA, L.S. Avaliação da atividade antimicrobiana do alho (Allium sativum Liliaceae) e de seu 
extrato aquoso. Rev. Bras. Pl. Med., Campinas, v.16, n.3, supl. I, p.679-684, 2014.

FRANCISQUINI, JDF; MARTINS, E; SILVA, PHF; SCHUCK, P; PERRONE, IT; CARVA LHO, A. F. Reação de Maillard: uma revisão. Rev. Inst. Laticínios Cândido Tostes, v. 72, n. 1, p. 48-57, 2017

GEORGÉ, S., BRAT, P., ALTER, P., \& AMIOT, M. J. Rapid determination of polyphenols and vitamin $\mathbf{C}$ in plant-derived products. Journal of Agricultural and Food Chemistry, v. 53, n. 5, p. 1370-1373, 2005. ISSN 0021-8561.

HERNÁNDEZ, A. M. \& PRIETO GONZÁLES, E. A. Plantas que contienen polifenoles. Revista Cubana de Investigaciones Biomedica, Ciudad de La Habana, v.18, n. 1, p. 12-14, 1999.

IBGE. Produção Agrícola municipal (PAM), Lavouras temporárias, 2018. Disponível em: http://www.ibge.gov/estatisticas/economicas/agricultura-e-pecuaria/9117-producaoagricola-municipal-culturas-temporarias-e- permanentes.html?=\&t=resultados. Acesso em: 22 de Jan. de 2020.

INSTITUTO ADOLFO LUTZ. Métodos Físico-Químicos para Análise de Alimentos. 4. ed. São Paulo: Instituto Adolfo Lutz, 2008.

INSTITUTO ADOLFO LUTZ. Normas Analíticas do Instituto Adolf Lutz, métodos químicos e físicos para análises de alimentos. 3. ed. V. 1. São Paulo, 1985.

JANG, E.-K; SEO, J.-H \& LEE, S.-P. Physiological activity and antioxidative effects of aged black garlic (Allium sativum L.) extract. Jornal Coreano de Ciência e Tecnologia de Alimentos. 40. 443-448, (2008).

KWAK, E. J. \& LIM, S. I. The effect of sugar, amino acid, metal ion, and NaCl on model Maillard reaction under pH control. Amino Acids 27, 85-90, 2004.

KIM, J. S.; KANG, O. J. \& GWEON, O. C. Comparison of phenolic acids and flavonoids in black garlic at diferente termal processing steps. J. Funct. Foods, 5, p. 80, (2013).

LEE, C. H.; CHEN, Y. T.; HSIEH, H. J.; CHEN, K-T.; CHEN, Y-A.; WU, J. T.; TSAI, M. S.; LIN, J. A.; HSIEH, C. W. Exploring epigallocatechin gallate impregnation to inhibit 5hydroxymethylfurfural formation and the effect on antioxidant ability of black garlic. LWT, Volume 117, 2020.

LEE, Y. M.; GWEON, O. C.; SEO, Y.J; IM, J.; KANG, M. J.; KIM, M. J.; KIM, J. I. Antioxidant effect of garlic and aged black garlic in animal model of type 2 diabetes mellitus. Nutr Res Pract.; 3 (2): 156-161; 2009.

LEMOS, A. R. Caracterização físico-química, bioquímica e avaliação da atividade antioxidante em genótipos de Urucueiros (Bixa orellana L.). Dissertação de mestrado - Universidade Estadual do Sudoeste da Bahia (UESB), 2008.

LI, Y.; XU, S-Y.; SUN, D-W. Preparation of garlic powder with high allicin content by using combined microwave-vacuum and vacuum drying as well as microencapsulation. Journal of Food Engineering, Volume 83, 2007.

LIU, Q.; LI, J.; KONG, B.; LI, P.; XIA, X. Physicochemical and antioxidant properties of Maillard reaction products formed by heating whey protein isolate and reducing sugars. International Journal of Dairy Technology, v. 67, n. 2, p. 220-228, 2014.

LU, X.; LI, N.; QIAO, X.; QIU, Z.; LIU, P. Composition analysis and antioxidant properties of black garlic extract Journal of Food and Drug Análise, 25, pp. 340 - 349, (2017)

LU, X.; LI, N.; QIAO, X.; QIU, Z.; LIU, P. Effects of thermal treatment on polysaccharide degradation during black garlic processing. Lebensmittel-Wissenschaft und Technologie- Food Science and Technology, 95, pp. 223-229, (2018).

MALDONADE, I. R. \& MACHADO, E. R. Alho negro: características e benefícios à saúde. Embrapa Hortaliças - Artigo de divulgação na mídia (INFOTECA-E). Brasília, DF, p. 2833, maio 2016.

NAJMAN, K.; SADOWSKA, A.; HALLMANN, E. Influence of Thermal Processing on the 
Bioactive, Antioxidant, and Physicochemical Properties of Conventional and Organic Agriculture Black Garlic (Allium sativum L.). Appl. Sci., 10 (23), 8638, 2020.

NODA, Y.; ASADA, C.; SASAKI, C.; HASHIMOTO, S.; NAKAMURA, Y. Extraction method for increasing antioxidant activity of raw garlic using steam explosion. Biochemical Engineering Journal, Volume 73, 2013.

NOVO, C. F. Atividade antioxidante do alho (Allium sativum L.) quando submetido a diferentes processos tecnológicos. Dissertação de mestrado. Universidade Fernando Pessoa. Porto, 2018.

PEREIRA, C. C.; SEGALA, G. D.; SÁ, M. M. M.; SASAKI, M. S.; REZENDE, R. S. A.; FELICIANO, P. O.; NETO, R. S. Processamento de alho (allium sativum) para a produção de alho negro e utilização em preparações gastronômicas. XXII Encontro Latino Americano de Iniciação Científica, XVIII Encontro Latino Americano de PósGraduação e VIII Encontro de Iniciação à Docência - Universidade do Vale do Paraíba. 2018.

PIEKARSKI, P. Análise nutricional e fitoquímica de frutos da Morus nigra L. (Dissertação de Mestrado) - Universidade Federal do Paraná, Curitiba, 2013.

PIRES, L. S. ALHO NEGRO E CEBOLA NEGRA: determinação e avaliação de parâmetros de processamento, propriedades físico-químicas e termodinâmicas. $171 \mathrm{f}$. Tese (doutorado) - Universidade Estadual Paulista Julio de Mesquita Filho, Instituto de Biociências, Letras e Ciências Exatas, 2020.

PIRES, L. S. \& MAURO, M. A. ALHO NEGRO: DESENVOLVIMENTO DA COR E FORMAÇÃO DE AÇÚCARES REDUTORES. XXV Congresso Brasileiro de Ciência e Tecnologia de Alimentos e X CIGR Section IV Internacional Technical Symposium Fundação de Apoio da Universidade Federal do Rio Grande Sul. 2016.

PIRES, L. S. Processamento do alho negro. 102 f. Dissertação (mestrado) - Universidade Estadual Paulista Julio de Mesquita Filho, Instituto de Biociências, Letras e Ciências Exatas, 2014.

RE, R.; PELLEGRINI, N.; PROTEGGENTE, A.; PANNALA, A.; YANG, M.; RICE-EVANS, C. Antioxidant activity applying an improved abts radical cation decolorization assay. Free Radical Biology \& Medicine, Vol. 26, Nos. 9/10, pp. 1231-1237, 1999.

RÍOS-RÍOS, K.L.; MONTILLA, A.; OLANO, A.; VILLAMIEL; M. Physicochemical changes and sensorial properties during black garlic elaboration: A review. Trends in Food Science \& Technology, 88 (2019), pp. 459-467. Disponível em: http://10.1016/j.tifs.2019.04.016.

RODRIGUEZ, A.; CUFFIA, F.; PIAGENTINI, A.; LEMA, P.; PANIZZOLO, L. A.; ROZYCKI, S. D. Study of the browning and gelation kinetics in a concentrated sheep milk and sucrose system. International Journal of Dairy Technology, v. 69, p. 1-7, 2016.

SANTOS, I. S. N. Avaliação do potencial antioxidante dos compostos fenólicos de extractos de plantas da flora portuguesa. Trabalho de Conclusão de Curso (Ciências Farmacêuticas). Universidade Fernando Pessoa, Faculdade de Ciência da Saúde - Porto, 2010.

SANTHOSHA, S. G.; JAMUNA, P.; PRABHAVATHI, S. N. Bioactive components of garlic and their physiological role in health maintenance: $A$ review. Food Bioscience, 3 (2013), p. 59-74.

SAVI, A. otimização do processo de extração de compostos Bioativos de folha de Jambo (Syzygium malaccense). Trabalho de conclusão de curso (graduação) - Universidade Tecnológica Federal do Paraná (UTFPR), Campus Pato Branco, 2015.

SHIBAO, J. \& BASTOS, D. H. M. Produtos da reação de Maillard em alimentos: implicações para a saúde. Rev. Nutr., Campinas, v. 24, n. 6, p. 895-904, Dez. 2011. 
SHIBAO, J. Avaliação do teor de Produtos da Reação Maillard (PRM) em cereais matinais e café. 101 f. Dissertação (mestrado) - Universidade de São Paulo Faculdade de Saúde Pública, 2010.

SILVA, A. B. Otimização da metodologia para produção de pastas de alho negro pela adição de mel e glutamato monossódico. 2013. 48 f. Trabalho de Conclusão de Curso. (Engenharia de Alimentos), Universidade Tecnológica Federal do Paraná - Campo Mourão, 2013.

SILVA, M. L. C., COSTA, R. S., SANTANA, A. S., KOBLITZ, M. G. B. (2010). Compostos fenólicos, carotenóides e atividade antioxidante em produtos vegetais. Semina: Ciências Agrárias. 31 (3).

SILVA, K. M. P.; LEITE, R. S. A.; RESENDE, F.V. Cultivares de alho comum para sistemas orgânicos de produção nas condições do cerrado. Horticultura Brasileira 26: S1764S1768. 2008.

SINGLETON, V.; ROSSI, J. A. Colorimetry of total phenolics with phosphomolybdicphosphotungstic acid reagents. American journal of Enology and Viticulture, v. 16, n. 3, p. 144-158, 1965. ISSN 0002-9254.

TACO - Tabela Brasileira de Composição de Alimentos / NEPA - UNICAMP. 4. ed. rev. e ampl. Campinas: NEPAUNICAMP, 2011. p.30 e 33.

TBCA - Tabela Brasileira de Composição de Alimentos. Universidade de São Paulo (USP). Food Research Center (FoRC). Versão 7.1. São Paulo, 2020. Acesso em: 11 de Dez. de 2020. Disponível em: http://www.fcf.usp.br/tbca.

TOLEDANO-MEDINA, M. A.; PEREZ-APARICIO, J.; MORENO-ROJAS, R.; MERINASAMO, T. Evolution of some physicochemical and antioxidant properties of black garlic whole bulbs and peeled cloves. Food Chem., 199, pp. 135-139, (2016).

VIEIRA, R. L. Caracterização genética dos acessos do bando ativo de germoplasta de alho (Allium sativum L.) de Santa Catarina. Dissertação (Mestrado em Recursos Genéticos Vegetais) - Centro de Ciências Agrárias, Universidade Federal de Santa Catarina, Florianópolis, 2004.

WANG, W. \& SUN, Y. B. In vitro and in vivo antioxidant activities of polyphenol extracted from black garlic. Food Sci. Technol, Campinas, v. 37, n. 4, p. 681-685, Dez. 2017.

YILMAZ, Y. \& TOLEDO, R. Antioxidant activity of water-soluble maillard reaction products. Food chem 93, 273 - 278 (2005).

ZHANG, X.; LI, N.; LU, X.; LIU, P.; QIAO, X. Effects of temperature on the quality of black garlic. Journal of the Science of Food and Agriculture , 96 ( 7 ), pp. 2366 - 2372, 2016.

ZOU, Y.; ZHAO, M.; YANG, K.; LIN, L.; WANG, Y. Enrichment of antioxidants in black garlic juice using macroporous resins and their protective effects on oxidationdamaged human erythrocytes. J. Chromatogr. B., 1060, p. 443-450, 2017. 